Check for updates

Cite this: Phys. Chem. Chem. Phys., 2017, 19, 25478

Received 28th June 2017

Accepted 24th August 2017

DOI: $10.1039 / c 7 c p 04357 k$

rsc.li/pccp

\title{
Role of intermolecular charge delocalization and its dimensionality in efficient band-like electron transport in crystalline 2,5-difluoro-7,7,8,8- tetracyanoquinodimethane $\left(\mathrm{F}_{2}-\mathrm{TCNQ}\right) \dagger$
}

\author{
Andrey Yu. Sosorev (D)
}

\begin{abstract}
Theoretical understanding of charge transport in organic semiconductors is exclusively important for organic electronics, but still remains a subject of debate. The recently discovered record-high band-like electron mobility in single crystals of 2,5-difluoro-7,7,8,8-tetracyanoquinodimethane $\left(F_{2}-T C N Q\right)$ is challenging from the theoretical viewpoint. First, the very small size of the $F_{2}-T C N Q$ molecule implies high reorganization energy that seems incompatible with efficient charge transport. Second, it is not clear why the crystals of a similar compound, 7,7,8,8-tetracyanoquinodimethane (TCNQ), show an inefficient hopping electron transport mechanism. To address these issues, we apply DFT and QM/MM calculations to the $F_{n}-\mathrm{TCNQ}(n=0,2,4)$ crystal series. We show that multidimensional intermolecular charge delocalization is of key importance for efficient charge transport in materials consisting of smallsized molecules, and commonly used guidelines for the search for high-mobility organic semiconductors are to be corrected.
\end{abstract}

\section{Introduction}

Organic electronics requires materials with efficient charge transport, i.e. high charge mobility $\mu$. However, only several organic semiconductors (OSCs) with $\mu>1 \mathrm{~cm}^{2}(\mathrm{~V} \mathrm{~s})^{-1}$ have been discovered. ${ }^{1}$ It is commonly considered that charge transport in OSCs is determined by the interplay of two factors. ${ }^{2-4}$ The first factor is charge carrier delocalization over several molecules ("sites") due to the electronic coupling between them; this coupling is commonly described with transfer integrals $J$. The opposite factor is charge localization at one site due to the electron-phonon coupling. Local contribution to the electronphonon coupling stems from the site energy change after charge transfer and is associated with the reorganization energy $\lambda$. If $\lambda \gg J$, which is typical for OSCs, charge localization occurs, and incoherent hopping charge transport mechanism with low $\mu$ takes place. Otherwise, delocalization can enable coherent band-like charge transport with high $\mu^{2,3}$ In high-mobility OSCs, non-local electron-phonon coupling related to the modulation of $J$ by vibrations plays a significant role. ${ }^{3}$

To improve the charge mobility and reach the efficient bandlike charge transport regime, $J$ values are to be increased, and $\lambda$ is to be decreased. The $J$ values are governed by the crystal

Faculty of Physics and International Laser Center, M.V. Lomonosov Moscow State University, Moscow 119991, Russia.E-mail: sosorev@physics.msu.ru

$\dagger$ Electronic supplementary information (ESI) available. See DOI: 10.1039/c7cp04357k structure since they are very sensitive to the relative positions of the molecules. ${ }^{4}$ The largest $J$ values are typically observed for parallel molecules with strongly overlapping $\pi$-conjugated systems (in the so-called $\pi$-stacks). ${ }^{5}$ Reorganization energy $\lambda$ consists of the intramolecular contribution, $\lambda_{i}$, related to the changes in molecular geometry after charge transfer, and the outer-sphere one, $\lambda_{\mathrm{o}}$, related to the polarization of the environment. It is commonly assumed that the former contribution dominates in OSCs, i.e. $\lambda \approx \lambda_{\mathrm{i}}{ }^{2}$. The $\lambda$ value can be reduced via enlarging the molecular conjugated core and increasing its rigidity, that's why most of the developed high-mobility OSCs consist of condensed aromatic molecules with prolonged $\pi$-conjugated systems. ${ }^{2,5}$ Recently, it was noticed that $\lambda$ in the crystal can be lower than that for the isolated molecule. First, neighboring molecules restrict the geometry reorganization of the given molecule after charge transfer. ${ }^{6,7}$ Second, charge carrier delocalization over adjacent molecules reduces the excess charge density on each of them and inhibits their geometry relaxation after charge transfer. ${ }^{8}$ The impact of the crystal environment on $\lambda$ can be essential for charge transport and should be considered when estimating $\lambda$; however, it is usually neglected.

A rigorous theoretical description of the charge transport in OSCs is still missing. ${ }^{3,9}$ An investigation of the OSC series consisting of structurally close molecules can improve the understanding of this process. A perfect example of such series is the crystals of fluorinated derivatives of 7,7,8,8-tetracyanoquinodimethane (TCNQ). As reported in ref. 10, one of them, 
2,5-difluoro-7,7,8,8-tetracyanoquinodimethane ( $\left.\mathrm{F}_{2}-\mathrm{TCNQ}\right)$, exhibits extremely high electron mobility $\left(\mu_{\mathrm{e}}=7 \mathrm{~cm}^{2}(\mathrm{~V} \mathrm{~s})^{-1}\right.$ at room temperature), while the corresponding quantity in its counterparts, TCNQ and $\mathrm{F}_{4}$-TCNQ, is two orders of magnitude lower $\left(\mu_{\mathrm{e}}=0.1\right.$ and $0.2 \mathrm{~cm}^{2}(\mathrm{~V} \mathrm{~s})^{-1}$, respectively). Moreover, when the temperature decreases, $\mu_{\mathrm{e}}$ in crystalline $\mathrm{F}_{2}$-TCNQ rises up to $c a$. $15 \mathrm{~cm}^{2}(\mathrm{~V} \mathrm{~s})^{-1}$ at $200 \mathrm{~K}$ and further up to $c a .25 \mathrm{~cm}^{2}(\mathrm{~V} \mathrm{~s})^{-1}$ at $150 \mathrm{~K}$. In contrast, in TCNQ and $\mathrm{F}_{4}$-TCNQ, $\mu_{\mathrm{e}}$ decreases with cooling, reaching $c a$. 0.01 and $0.03 \mathrm{~cm}^{2}(\mathrm{~V} \mathrm{~s})^{-1}$, respectively, at $200 \mathrm{~K}$. Therefore, the charge transport mechanism is qualitatively different in these compounds: it is band-like in $\mathrm{F}_{2}$-TCNQ indicating pronounced electron delocalization, and hopping in TCNQ and $\mathrm{F}_{4}$-TCNQ, indicating localized charges. In ref. 10, record-high electron mobility in $\mathrm{F}_{2}$-TCNQ was attributed to strong electronic coupling between the molecules resulting in a wide conduction band, and low $\mu_{\mathrm{e}}$ in crystalline $\mathrm{F}_{4}$-TCNQ was explained by a narrow conduction band (i.e. low $J$ ). ${ }^{10}$ However, the $c a$. 70-fold difference in room-temperature $\mu_{\mathrm{e}}$ between $\mathrm{F}_{2}$-TCNQ and TCNQ remained unexplained since the bandwidths were found to be comparable for these materials. Later, in ref. 11, this difference was attributed to strong non-local electron-phonon coupling in crystalline TCNQ, which was deduced from the two-fold decrease in the lowest vibrational frequency $\omega_{0}$ that can affect $\mu_{\mathrm{e}}$ dramatically. $^{3}$

However, two important issues remain open. First, $\mathrm{F}_{2}$-TCNQ molecules are very small as compared to typical OSCs (e.g. rubrene or pentacene) and are expected to possess strong local electron-phonon interaction (large $\lambda$ ) due to significant changes in molecular geometry after charge transfer. ${ }^{12}$ A large $\lambda$ favors charge localization that contradicts the observed efficient bandlike transport in $\mathrm{F}_{2}$-TCNQ. ${ }^{3,4}$ Second, although the different $\omega_{0}$ explains the difference in room-temperature $\mu_{\mathrm{e}}$ between the $\mathrm{F}_{2}$-TCNQ and TCNQ crystals, ${ }^{11}$ it does not explain the qualitatively different charge transport mechanism for these materials (bandlike $v s$. hopping). In addition, the $\omega_{0}$ difference between $\mathrm{F}_{2}$-TCNQ and TCNQ reduces with cooling (see the $\mathrm{ESI} \dagger$ to ref. 11), which should only smoothen out the difference in $\mu_{\mathrm{e}}$ for these materials, in contradiction with the observed increase of the latter up to $c a$. 1500 times at $200 \mathrm{~K}^{10}$ Therefore, it appears that non-local electronphonon interaction is not the source of qualitatively different charge transport in $\mathrm{F}_{2}$-TCNQ and TCNQ.

In this study, we investigate the electron transport in the $\mathrm{F}_{n}$-TCNQ ( $\left.n=0,2,4\right)$ crystal series using DFT and QM/MM approaches. Two abovementioned issues are addressed: (i) how efficient charge transport can occur in crystalline $\mathrm{F}_{2}$-TCNQ despite the small size of its molecules, and (ii) why electron transport is band-like in crystalline $\mathrm{F}_{2}$-TCNQ but hopping-like in crystalline TCNQ, with three orders of magnitude difference in $\mu_{\mathrm{e}}$ at $200 \mathrm{~K}$. We show that while $\lambda$ is large in the $\mathrm{F}_{n}$-TCNQ isolated molecules, in the TCNQ and $F_{2}$ TCNQ crystals it is strongly reduced due to intermolecular charge delocalization, making efficient charge transport possible. To address the qualitatively different transport mechanism in $\mathrm{F}_{2}$-TCNQ and TCNQ, we analyze $J$ along different directions and find that charge delocalization is three-dimensional in the former material but (quasi) one-dimensional in the latter. Enhanced dimensionality of intermolecular charge delocalization in $\mathrm{F}_{2}$-TCNQ is shown to be more resistant to the electron-phonon coupling and defects, explaining band-like charge transport in this material. Our findings force us to suggest corrections to the current guidelines for the search for high-mobility OSCs.

\section{Computational}

Crystal structures were obtained from the X-ray data. ${ }^{10}$ One molecule (A) and its selected nearest-neighbors are depicted in Fig. 1a for the TCNQ crystal, and possible charge transfer pathways are shown with arrows and labeled by the dimer types (see below).

The transfer integrals, Js, were calculated using a homewritten code based on the dimer projection method (DIPRO). ${ }^{13-15}$ To estimate this quantity for a pair (dimer) of molecules (monomers) A and B depicted in Fig. 1a, $J_{\mathrm{AB}}$, the wavefunctions of these molecules were approximated by their lowest unoccupied molecular orbitals (LUMOs), $\varphi_{\mathrm{A}}$ and $\varphi_{\mathrm{B}}$. The latter were written on the basis of the dimer molecular orbitals $\varphi_{i}$ :

$$
\begin{aligned}
J_{\mathrm{AB}} & =\left\langle\varphi_{\mathrm{A}}|H| \varphi_{\mathrm{B}}\right\rangle=\sum_{i} \sum_{j}\left\langle\varphi_{\mathrm{A}} \mid \varphi_{i}\right\rangle\left\langle\varphi_{i}|H| \varphi_{j}\right\rangle\left\langle\varphi_{j} \mid \varphi_{\mathrm{B}}\right\rangle \\
& \approx \sum_{i}\left\langle\varphi_{\mathrm{A}} \mid \varphi_{i}\right\rangle E_{i}\left\langle\varphi_{i} \mid \varphi_{\mathrm{B}}\right\rangle
\end{aligned}
$$

where $E_{i}$ are the energies of $\varphi_{i}$. Projections of monomer LUMOS on dimer orbitals, $\left\langle\phi_{\mathrm{A}} \mid \phi_{i}\right\rangle$ and $\left\langle\phi_{i} \mid \phi_{\mathrm{B}}\right\rangle$, were calculated from the corresponding coefficients on the basis of atomic orbitals. The energy-splitting-in-dimer (ESID) method, which treats $J$ as the half of the energy difference between the dimer LUMO and LUMO+1, was used to verify our realization of the DIPRO approach. The $J$ values for the two methods coincided within $10 \%$ accuracy, where the ESID method was applicable (see ESI, $\dagger$ Section S1).

Reorganization energy $\lambda$ was approximated by $\lambda_{\mathrm{i}}$ that is typically considered much larger than $\lambda_{\mathrm{o}}$ in OSCs. ${ }^{2}$ The $\lambda$ values were calculated according to the 4-point scheme ${ }^{4}$ illustrated in Fig. 1b. In this approach, the energies of the molecule in 4 states are required: neutral state in its optimized geometry ( $\mathrm{N}$ state in Fig. 1b), neutral state in the optimized geometry of the charged state $\left(\mathrm{N}^{*}\right)$, charged state in its optimized geometry (C), and charged state in the geometry of the neutral state $\left(\mathrm{C}^{*}\right)$. The energy difference between the former two states, $\lambda_{1}=E_{\mathrm{N}}{ }^{*}-$ $E_{\mathrm{N}}$, describes the energy relaxation of the molecule that has lost the charge carrier, while the energy difference between the latter two states, $\lambda_{2}=E_{\mathrm{C}}{ }^{*}-E_{\mathrm{C}}$, describes the energy relaxation of the molecule that has accepted the charge. The total reorganization energy is:

$$
\lambda=\lambda_{1}+\lambda_{2}=\left(E_{\mathrm{N}}^{*}-E_{\mathrm{N}}\right)+\left(E_{\mathrm{C}^{*}}^{*}-E_{\mathrm{C}}\right)
$$

The impact of the crystal environment on $\lambda$ can be accounted by the calculation of the abovementioned energies for a system containing a given molecule and its frozen-geometry neighbors. In practice, this task cannot be solved using DFT or other quantum-chemical approaches. Nevertheless, it can be addressed using a combined quantum mechanical/molecular mechanical method (QM/MM) performing quantum mechanical (QM) calculations, e.g. DFT, for the given molecule, and classical 
a)

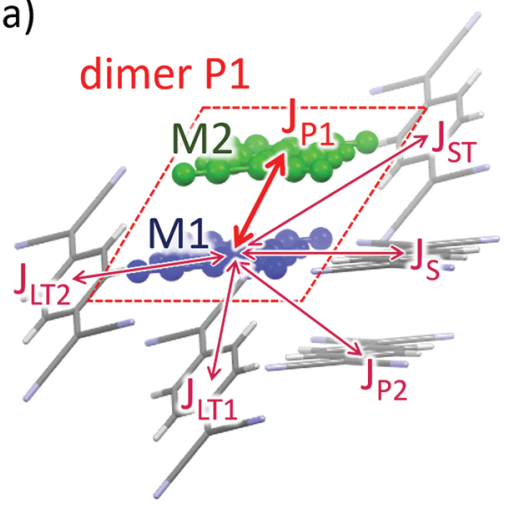

b)

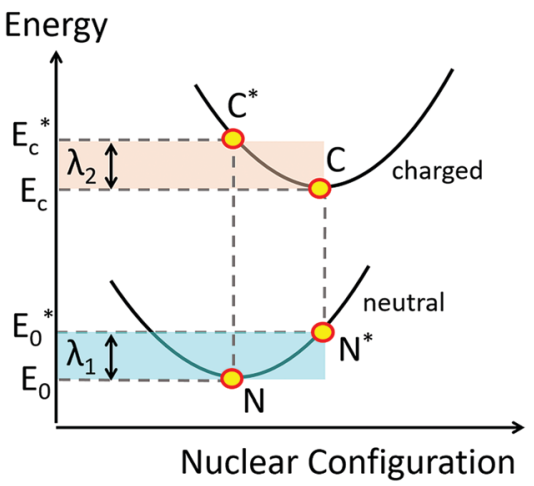

Fig. 1 (a) TCNQ molecule and its environment in the crystal. The probable pathways for charge transfer from the central molecule A (blue) are shown with red arrows and labeled with the corresponding transfer integrals. One of the dimers, P1, is highlighted with the dashed line. (b) Illustration of the four-point scheme for the reorganization energy calculation. Potential energy curves for the neutral and charged molecules are shown with bottom and top parabolas, respectively. Yellow circles denote the states for which energy is to be calculated.

molecular mechanics (MM) for its environment. ${ }^{16}$ To estimate the impact of the charge delocalization, we propose an original approach that is described in the Results section.

For charge mobility estimation within the hopping model, charge transfer rates from a given molecule A to each of its nearest neighbors (e.g. molecule B) were calculated using the Marcus formula: ${ }^{17}$

$$
k_{\mathrm{AB}}=\frac{2 \pi}{\hbar} J_{\mathrm{AB}}{ }^{2} \frac{1}{\sqrt{4 \pi \lambda k T}} \exp \left(-\frac{(\Delta E-\lambda)^{2}}{4 \lambda k T}\right),
$$

where $\hbar$ is the reduced Planck constant, $k$ is the Boltzmann constant, $T$ is the absolute temperature, and $\Delta E$ is the electron energy difference between the initial and final sites $(\Delta E=0$ if the molecules are similar). Charge mobility was calculated using eqn (3) using the Einstein-Smoluchowski relation (see e.g. ref. 18). Although this approach is simplified and describes the hopping charge transport regime, it is frequently used to estimate charge mobility in organic semiconductors, ${ }^{9,18-21}$ and its predictions show a qualitative correlation with the experimental data even for highmobility OSCs with band-like mobility. ${ }^{9,18,21}$

All DFT calculations were performed using the B3LYP functional. For the $J$ calculation, the $6-31 \mathrm{~g}^{*}$ basis set was used, and $\lambda$ estimation was conducted using the $6-31+\mathrm{g}^{* *}$ basis set for isolated molecules and the 6-31g basis set when considering the crystal environment. The impact of the basis set on $\lambda$ and $J$ is discussed in ESI, $\dagger$ Section S2. GAMESS package ${ }^{22,23}$ was used to calculate $J$ and $\lambda$ for isolated molecules, and NWChem package ${ }^{24}$ was used for $\mathrm{QM} / \mathrm{MM}$ estimation of $\lambda$ in crystals. For QM/MM calculations, mechanic embedding was used, and molecular mechanics was performed using the Amber force field.

\section{Results}

\section{Molecular packing and electron transfer integrals}

Fig. 2 shows the structures of the investigated crystals according to the X-ray data ${ }^{10}$ with transport pathways deduced from the calculated $J$ values.
The TCNQ crystal can be considered to consist of onedimensional (1D) $\pi$-stacks of parallel and slightly shifted molecules with significant overlapping of their $\pi$-conjugated systems $(\pi-\pi$ overlapping) providing large $J=69 \mathrm{meV}$. The dimer configuration in the stack is denominated below as the "parallel" P1-dimer. Stacks with similar orientations of molecular planes form twodimensional (2D) "slabs" corresponding to the (002) crystal plane. The slab is depicted in Fig. 2a. Within the slab, in addition to P1-dimers, "parallel" P2- and "side" S-dimers are observed (see the corresponding directions in Fig. 2a). In P2-dimers, the molecules are parallel like in P1-dimers, but a large shift along the short molecular axis reduces $\pi-\pi$ overlapping resulting in a low $J=14 \mathrm{meV}$. S-Dimers also show a low $J=10 \mathrm{meV}$ since molecules in them interact via $\mathrm{CH} \cdots \mathrm{N}$ contacts that do not provide considerable $\pi-\pi$ overlapping. In adjacent slabs, the molecular planes are tilted with respect to each other (Fig. 2b), like in the herringbone packing motif. Three types of dimers can provide charge transport between the slabs: two "longitudinal, tilted" dimers LT1 and LT2, and "side, tilted" ST-dimers. In the LT1-dimer configuration, the molecules interact via $\pi$-electron-rich dicyanomethylidene (DCMI) groups resulting in a moderate $J=32 \mathrm{meV}$, and for ST and LT2-dimers, negligible $J=7$ and $1 \mathrm{meV}$ stem from very weak $\pi-\pi$ overlapping.

The $\mathrm{F}_{2}$-TCNQ crystal can be also considered to be formed of 2D slabs of parallel molecules. The slab corresponds to the (001) crystal plane and is shown in Fig. 2c. It is determined by the $\pi$-stacking directions P1 and P2, in which the molecules are slightly shifted with respect to each other providing a large $J=50 \mathrm{meV}$. As in TCNQ crystals, S1-dimers consisting of molecules interacting via side $\mathrm{CH} \cdots \mathrm{N}$ contacts show a low $J=11 \mathrm{meV}$. Unlike TCNQ, molecular planes in adjacent slabs are not tilted but are parallel (Fig. 2d) due to the specific crystal packing with only one molecule in the reduced cell. ${ }^{10,11}$ Adjacent molecules from different slabs form "longitudinal" $\mathrm{L}$ - and "parallel-longitudinal" PL-dimers, in which the molecules interact via DCMI groups. Interestingly, PL-dimers show a large $J=69 \mathrm{meV}$ despite a significant shift of the molecules; this can 
TCNQ

a) slab

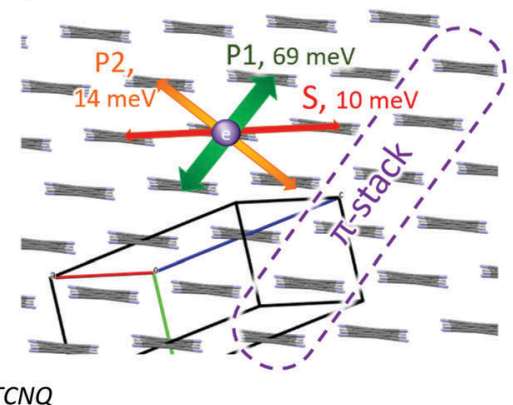

$F_{2}$-TCNQ

c) slab

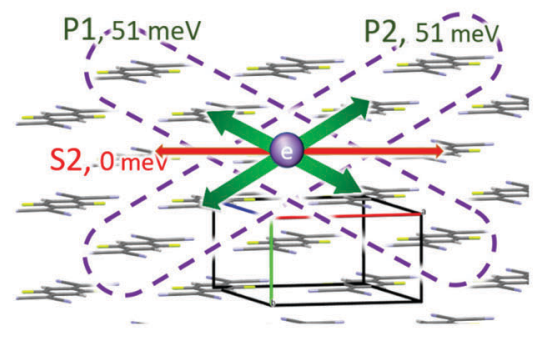

$F_{4}-T C N Q$

e) slab

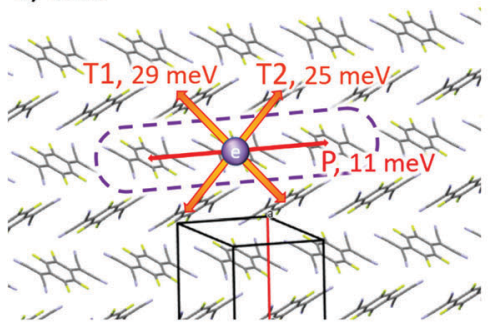

b) side view

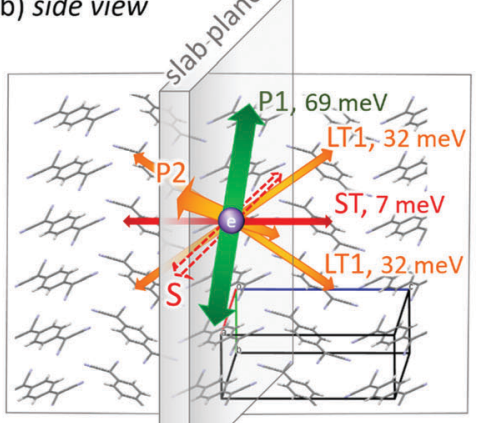

d) side view
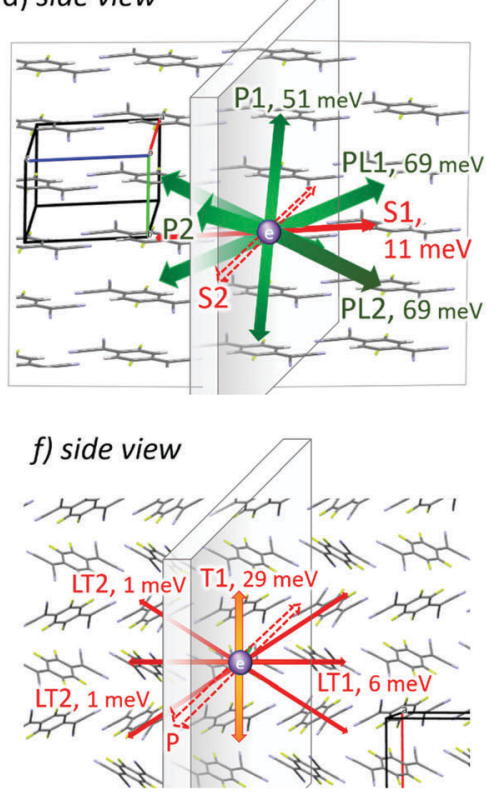

Fig. 2 Crystal structures of TCNQ (top), $F_{2}-T C N Q$ (middle) and $F_{4}-T C N Q$ (bottom) with charge transport directions shown by arrows. Left panels depict slab planes (see in the text): (a) the (002) plane of the TCNQ crystal, (c) the (001) plane of $F_{2}-T N C Q$, and (e) the (002) plane of $F_{4}-T C N Q$. Violet dashed areas highlight $\pi$-stacks. Right panels ( $b, d$ and f) show the planes approximately normal to the slabs in crystalline $T C N Q, F_{2}-T C N Q$, and $F_{4}-T C N Q$, respectively; the slabs are shown in grey. Labels indicate dimer types and $J$ values for various charge transfer directions. The color and thickness of the arrows designate $J$ magnitude: thick green arrows depict pathways with $J \geq 2 k T$, thin red arrows indicate $J<k T / 2$, and orange arrows correspond to $k T / 2 \leq J<2 k T$.

be attributed to the proximity of their $\pi$-electron-rich DCMI groups resulting in strong $\pi-\pi$ overlapping. Negligible $J=4 \mathrm{meV}$ in L-dimers and $J=0$ in S2-dimers result from weak $\pi-\pi$ overlapping because of a large intermolecular distance.

In crystalline $\mathrm{F}_{4}$-TCNQ, $\pi$-stacking takes place along the $\mathrm{P}$ direction, but the molecules in the corresponding P-dimers are strongly shifted along both molecular axes resulting in poor $\pi-\pi$ overlapping and a low $J=11 \mathrm{meV}$. Unlike the first two crystals, the molecular planes in any adjacent $\pi$-stacks are tilted with respect to each other. The slab definition is therefore arbitrary, and we chose the (002) crystal plane as a slab (Fig. 2e). The dimer types observed within the slab are P-dimers and "transverse", T-dimers $(J=29$ and $25 \mathrm{meV})$. LT-dimers of several types connect different slabs, possessing $J=1$ and $6 \mathrm{meV}$ (Fig. 2f).

Fig. 3a collates the $J$ values for various dimer configurations in the considered crystals. The maximal transfer integrals $J_{\max }$ in TCNQ and $\mathrm{F}_{2}$-TCNQ are equal (69 $\mathrm{meV}$ ) and rather large (close to $c a$. $70 \mathrm{meV}$ for pentacene and rubrene ${ }^{1}$ ), while in $\mathrm{F}_{4^{-}}$ TCNQ $J_{\max }$ is twice lower (29 meV). In TCNQ and $\mathrm{F}_{2}$-TCNQ, large $J$ values are observed in the dimers of parallel molecules, namely in P1-dimers in TCNQ, and PL-, P1- and P2-dimers in $\mathrm{F}_{2}$-TCNQ. This is in line with the common assumption of the essential role of $\pi$-stacking in charge transport. ${ }^{5}$ The dimers in which the molecular planes are tilted with respect to each other, namely T- and LT-dimers, show $J$ values below $35 \mathrm{meV}$ because of reduced $\pi-\pi$ overlapping for non-parallel molecules. Nevertheless, T-dimers contribute significantly to charge transport in crystalline $\mathrm{F}_{4}$-TCNQ since P-dimers in this material possess a low $J$.

\section{Reorganization energy and electron mobility within the standard hopping model}

The calculated intramolecular reorganization energies $\lambda$ of the isolated TCNQ, $\mathrm{F}_{2}$-TCNQ and $\mathrm{F}_{4}$-TCNQ molecules are very close 


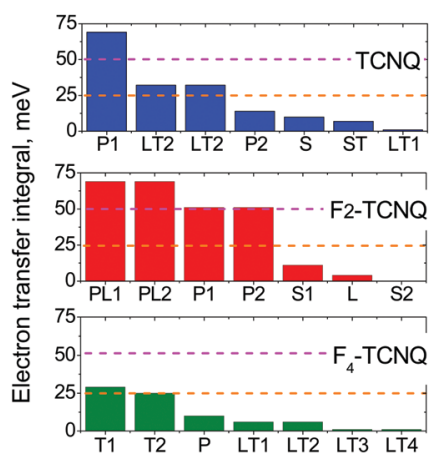

Fig. 3 Electron transfer integrals for various dimer configurations in the $\mathrm{F}_{n}$-TCNQ crystals (a) and electron mobility estimated within the hopping model based on the Marcus theory (b).

and equal to 250,258 and $248 \mathrm{meV}$, respectively. These values are rather large (compared to $\lambda=134 \mathrm{meV}$ for rubrene and $91 \mathrm{meV}$ for pentacene ${ }^{1}$ ) and stem from the small size of $\mathrm{F}_{n}$-TCNQ molecules inducing strong changes in molecular geometry after charge transfer, ${ }^{12}$ in accordance with our concerns mentioned in the Introduction.

The electron mobilities $\mu_{\mathrm{e}}$ in the TCNQ, $\mathrm{F}_{2}$-TCNQ and $\mathrm{F}_{4}$-TCNQ crystals estimated within the hopping model based on eqn (3) are $0.25,0.54,0.05 \mathrm{~cm}^{2}(\mathrm{~V} \mathrm{~s})^{-1}$, respectively (Fig. 3b). An order of magnitude lower $\mu$ for $\mathrm{F}_{4}$-TCNQ stems from 2.5 -fold lower $J_{\max }$ values (Fig. 3a), in correspondence with experimental data and calculations from ref. 10 . However, the calculated $\mu_{\mathrm{e}}$ for $F_{2}$-TCNQ is 13 times lower than the experimental one. Further, comparable $J$ and $\lambda$ values for $F_{2}$-TCNQ and TCNQ resulting in similar $\mu_{\mathrm{e}}$ values do not explain the qualitative experimental difference in charge transport mechanism between the two materials. These are actually the two issues raised in the Introduction: the common model did not resolve them. Therefore, an in-depth analysis is required, which is provided below.

\section{Impact of the crystal environment on reorganization energy}

The moderate calculated $\mu_{\mathrm{e}}$ value for $\mathrm{F}_{2}$-TCNQ stems mainly from high $\lambda$. To reconcile the contradiction between the calculated $\lambda$ and observed efficient charge transport in $\mathrm{F}_{2}$-TCNQ, it is natural to suggest that $\lambda$ in the $\mathrm{F}_{2}$-TCNQ crystal is lower than for the isolated molecule. As mentioned in the Introduction, the crystal environment can reduce $\lambda$ via two mechanisms: restriction of intramolecular motions (RIM) by surrounding molecules, and intermolecular charge delocalization. To estimate the impact of RIM, we applied a QM/MM-based approach described in ref. 6 and 7. One molecule was calculated at the quantum mechanical (QM) level (DFT, B3LYP/6-31g), while the environment that restricts its motion was described by frozen-geometry nearest neighbors (14 molecules in the considered crystals) using molecular mechanics (MM). The $\lambda$ value was then calculated according to eqn (2). The obtained $\lambda^{\text {RIM }}$ values for TCNQ, $\mathrm{F}_{2}$-TCNQ and $\mathrm{F}_{4}$-TCNQ are 237, 240 and $223 \mathrm{meV}$, correspondingly. These values are close to those for isolated molecules (237, 230 and $212 \mathrm{meV}$, respectively, at the same DFT level), and the $\lambda$ decrease due to RIM is not observed.
To estimate the impact of the intermolecular charge delocalization on $\lambda$, we propose the following original approach based on QM/MM. We consider systems of several molecules embedded into the crystal environment - dimer, trimer, tetramer, etc. The optimized geometries of these systems are obtained at the QM level considering the crystal environment (frozen-geometry nearest-neighbor molecules) described at the MM level. The reorganization energy considering delocalization, $\lambda^{\mathrm{del}}$, is estimated using eqn (2), with the only difference that the energies of $\mathrm{N}, \mathrm{N}^{*}, \mathrm{C}$ and $\mathrm{C}^{*}$ states are calculated for the dimer (trimer, tetramer, etc.) instead of a single molecule. To the best of our knowledge, QM/ MM was not used before to address the impact of the charge delocalization on $\lambda$.

Using this approach, we estimated $\lambda^{\mathrm{del}}$ values for the TCNQ and $\mathrm{F}_{2}$-TCNQ crystals, where large $J$ values can promote efficient charge delocalization. The $\lambda^{\mathrm{del}}$ was not calculated for the $\mathrm{F}_{4}$-TCNQ crystal since low $J$ values are not capable of providing substantial charge delocalization. Chart 1 illustrates the considered dimer configurations and their environment for TCNQ (P1, LT and P2 dimers) and $\mathrm{F}_{2}$-TCNQ (PL, P1 and $\mathrm{S}$ dimers). Trimers of $\mathrm{F}_{2}$-TCNQ molecules along the P1 and PL directions were regarded, as well as trimers and tetramers of TCNQ molecules along the P1 direction. Fig. 4 collates the calculated $\lambda^{\mathrm{del}}$ for dimers, trimers and tetramers in the crystal environment, as well as $\lambda$ and $\lambda^{\mathrm{RIM}}$. The $\lambda^{\mathrm{del}}$ values for dimers are about 1.5-2 times lower than the $\lambda$ and $\lambda^{\text {RIM }}$ values. Specifically, in the P1-dimer of $\mathrm{F}_{2}$-TCNQ, the reorganization energy becomes as low as $\lambda^{\mathrm{del}}=130 \mathrm{meV}$, i.e. its decrease as compared to $\lambda$ amounts $100 \mathrm{meV}$. In TCNQ, $\lambda_{\mathrm{i}}^{\text {del }}=145 \mathrm{meV}$ for the P1-dimer, which is $90 \mathrm{meV}$ lower than $\lambda$. The $\lambda^{\mathrm{del}}$ value decreases further for P1-trimers: it is just $95 \mathrm{meV}$ for $\mathrm{F}_{2}$-TCNQ and $100 \mathrm{meV}$ for TCNQ, which is close to the $\lambda$ value for the isolated pentacene molecule. ${ }^{1}$ For the TCNQ tetramer, $\lambda^{\text {del }}$ reaches an extremely low value of $c a .75 \mathrm{meV}$.

\section{Discussion}

The results presented above show that the reorganization energy $\lambda$ of the $\mathrm{F}_{n}$-TCNQ molecules is strongly reduced by the crystal environment due to charge delocalization. These results are in line with our recent findings reported in ref. 11, where intermolecular vibrations disrupting the delocalization were suggested to be detrimental for $\mu_{\mathrm{e}}$. The fact that the second factor, RIM, does not reduce $\lambda$ can be attributed to the small size of the considered molecules and their internal rigidity. Indeed, a significant $\lambda$ decrease due to RIM was observed in crystals of large and "soft" molecules. ${ }^{6,7}$

Our method for considering charge delocalization in the $\lambda$ calculation is a natural combination of the approach suggested in ref. 8 for the same purpose and the approach proposed in ref. 6 and 7 to account for RIM. The advance of our approach as compared to that reported in ref. 8 is in avoiding any artificial geometry constraints for the molecules over which the charge is delocalized. In our method, their geometry is naturally constrained by the interaction with (frozen-geometry) nearest neighbors, while in ref. 8 some of the degrees of freedom were fixed artificially. 


\section{TCNQ}

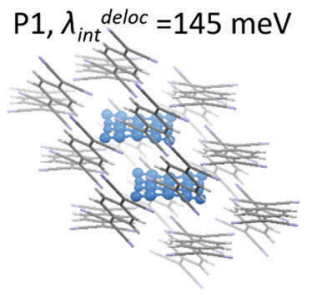

$\mathrm{F}_{2}$-TCNQ
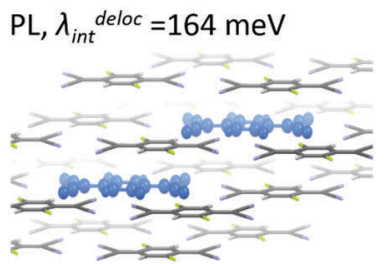

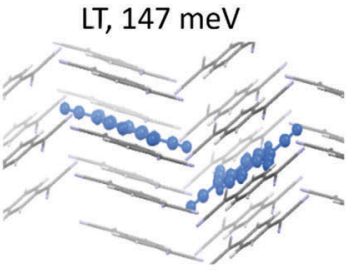

$\mathrm{P} 1,130 \mathrm{meV}$

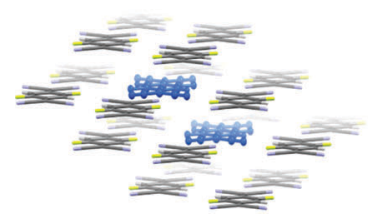

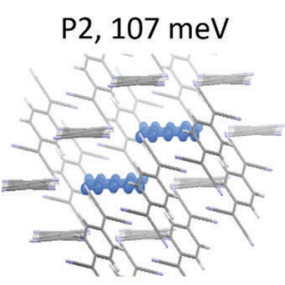

S, $97 \mathrm{meV}$

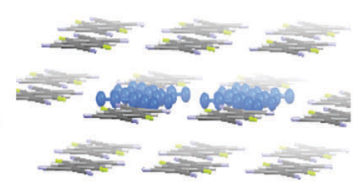

Chart $1 \mathrm{TCNQ}$ and $\mathrm{F}_{2}-\mathrm{TCNQ}$ dimers for which the impact of the intermolecular charge delocalization on the reorganization energy was addressed.

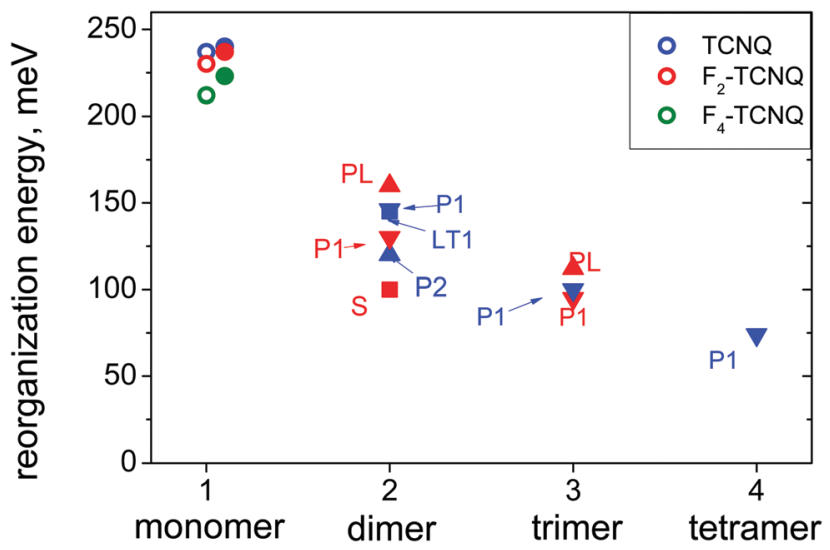

Fig. 4 Intramolecular reorganization energies in the $F_{n}-\mathrm{TCNQ}$ crystals. Open circles: isolated molecules, solid symbols: in the crystal environment. The dimer types are designated by labels and symbol shapes: up triangles denote PL-dimers, down triangles P1-dimers, squares S-dimers, and circles monomers.

Although our approach does not provide absolute values of $\lambda$ in the crystal (since DFT typically overestimates the extent of charge delocalization $^{8}$ ), it clearly illustrates a dramatic $\lambda$ decrease in crystalline $\mathrm{F}_{n}$-TCNQ due to intermolecular charge delocalization, and provides an opportunity to compare this effect in different materials.

One could suggest that the delocalization-induced decrease of $\lambda$ is related to $J$ : the larger the $J$, the more pronounced the delocalization, and probably the lower the $\lambda^{\text {del }}$. However, this is not observed: for instance, although the P1-dimer in TCNQ has a larger $J$ than in $\mathrm{F}_{2}$-TCNQ, it shows a higher $\lambda^{\text {del }}$. Moreover, even for different dimers of the same crystal, larger $J$ values do not cause lower $\lambda$ ( $c f$. P1- and PL-dimers in $\mathrm{F}_{2}$-TCNQ, or P1 and LT-dimers in TCNQ). We suggest that the key factors determining $\lambda^{\text {del }}$ are intermolecular vibrations that can have a large amplitude due to their weak force constant and modulate $J$ significantly thus disrupting the delocalization. ${ }^{3,25}$ Accordingly, these vibrations affect the energies of the dimers, i.e. contribute to $\lambda^{\text {del }}$. Parallel sliding along the molecular axis can have a very large amplitude ${ }^{26}$ and modulate $J$ strongly, ${ }^{27}$ which explains the larger $\lambda^{\text {del }}$ for the PL-dimer.

Reduction of $\lambda$ by the crystal environment explains the observed efficient charge transport in $\mathrm{F}_{2}$-TCNQ. Substituting $\lambda^{\text {del }}$ for P1-trimers instead of $\lambda$ in eqn (3) rises electron mobilities $\mu_{\mathrm{e}}$ in TCNQ and $\mathrm{F}_{2}$-TCNQ up to $\mu_{\mathrm{e}}^{\text {del }}=1.7$ and $4 \mathrm{~cm}^{2}(\mathrm{~V} \mathrm{~s})^{-1}$, respectively. The $\mu_{\mathrm{e}}^{\mathrm{del}}$ value for $\mathrm{F}_{2}$-TCNQ is much closer to the experimental one $\left(7 \mathrm{~cm}^{2}(\mathrm{~V} \mathrm{~s})^{-1}\right)$ compared to $\mu_{\mathrm{e}}=0.54 \mathrm{~cm}^{2}(\mathrm{~V} \mathrm{~s})^{-1}$ without considering delocalization, and the actual intermolecular delocalization length can exceed two molecules resulting in an even higher $\mu_{\mathrm{e}}^{\mathrm{del}}$. We therefore argue that the first issue concerning efficient charge transport in crystalline $\mathrm{F}_{2}$-TCNQ despite the small size of its molecules is reconciled.

However, the second challenge, namely the difference in transport mechanism between $\mathrm{F}_{2}$-TCNQ and TCNQ, remains unsolved. As we have shown above, in these materials $\lambda^{\text {del }}$ and $J_{\max }$ are similar, with $\lambda^{\text {del }} \sim J_{\max }$ that could enable band-like charge transport in both $\mathrm{F}_{2}$-TCNQ and TCNQ. ${ }^{28}$ Therefore, to explain the experimental data, it is natural to suggest that intermolecular charge delocalization is disrupted in TCNQ, but present in $\mathrm{F}_{2}$-TCNQ. Since delocalization/localization depends on the relationship between $J$ and $\lambda$, we analyze below the electron transfer integrals $J$ along different directions.

As can be deduced from Fig. $2 \mathrm{a}, \mathrm{b}$ and $3 \mathrm{a}$, in the TCNQ crystal, significant $J \geq 2 k T(k T=25 \mathrm{meV}$ at room temperature) are observed in only one direction, namely along the $\pi$-stack P1. Transfer integrals connecting the stacks within the slab (P2- and S-dimers) are lower than $k T$ and hence should be strongly modulated by thermal fluctuations, as well as $J$ values between the slabs (LT1) that are comparable to $k T$. We conclude that in the TCNQ crystal, efficient charge transport can occur only along the $\pi-\pi$ stacking direction (P1) at room temperature, i.e. is (quasi)1D. In contrast, in the $\mathrm{F}_{2}$-TCNQ crystal there are 4 directions with $J \geq 2 k T$ (Fig. 2c, d and 3a): two within the 
slab (P1 and P2), and two between different slabs (PL). These directions cover all the three space dimensions (see Fig. 2c and d). We thus conclude that the dimensionality of charge transport in $\mathrm{F}_{2}$-TCNQ is higher than in TCNQ, namely it is 3D instead of (quasi)1D.

To illustrate the role of charge delocalization dimensionality in charge transport, we use a simple one-electron tight-binding model. Assume a set of $\mathrm{N}$ sites arranged in a 1D, 2D or 3D lattice. A charge carrier, e.g. electron, can be either localized at one site or delocalized over several sites. Its wavefunction is $\psi=\sum_{i=1}^{N} c_{i} \varphi_{i}$, where $\varphi_{i}$ are the wavefunctions of the electron localized at $i$-th site and $c_{i}$ are the coefficients. The mechanism of localization is the decrease of the energy for the site occupied by the electron. This decrease corresponds to the polaron binding energy and equals $E_{\mathrm{p}}=\lambda / 2$; accordingly, larger $\lambda$ induces stronger localization. Following ref. 29, we assume that if the electron occupies the $i$-th site partially, the site energy decreases by $c_{i}^{4} \cdot \lambda / 2$ (proportional to the squared extra charge at the site, which is proportional to $c_{i}^{2}$ ). The energy of the state is

$$
E=\frac{1}{2} \sum_{\substack{j=1 \\ j \neq i}}^{N} \sum_{i=1}^{N} J_{i j} c_{i} c_{j}+\sum_{i=1}^{N} \varepsilon_{i} c_{i}^{2}-\sum_{i=1}^{N} c_{i}^{4} \lambda / 2
$$

In eqn (4), $J_{i j}$ are the transfer integrals between sites $i$ and $j$, and $\varepsilon_{i}$ are the energies of the sites. For simplicity, we set equal $\varepsilon_{i}=\varepsilon$ for all sites, and equal $J_{i j}=J$ for adjacent sites and $J_{i j}=0$ otherwise. We will consider the states in which charge carrier is homogeneously distributed over $m=p^{d}$ sites, where $p$ is an integer number and $d$ is the dimensionality of the system $(1,2$ or 3$)$. The restriction on $m$ makes the calculation simpler due to the symmetry of the resulting site population, and does not affect the results. The wavefunction of such state reads $\psi=\sum_{i=1}^{m} \frac{1}{\sqrt{m}} \varphi_{i}$, and its energy is

$$
E=m \varepsilon+J d\left(1-m^{-1 / d}\right)-\lambda / m
$$

The $E(m)$ dependence described by eqn (5) has a minimum at either $m=1$ or $m=\infty$ depending on the relation between $J, \lambda$ and $d$. In the former case, the charge is localized, while in the latter case it is delocalized. Comparison of the $E$ values for these two states yields the charge delocalization criterion: delocalization occurs if

$$
J>J_{\mathrm{cr}}, \quad J_{\mathrm{cr}}=\frac{\lambda}{2 d},
$$

This criterion is less strict for higher $d$, i.e. delocalization occurs more readily in the $3 \mathrm{D}$ case than in $2 \mathrm{D}$ or $1 \mathrm{D}$. Note that in eqn (6), $\lambda$ denotes the reorganization energy at a single site, i.e. without considering delocalization. From the calculated $\lambda$ (ca. $250 \mathrm{meV}$ ) and $J$ (see Fig. 3a), the delocalization criterion is fulfilled in $\mathrm{F}_{2}$-TCNQ for all the three spatial dimensions $(d=3$, $J_{\text {cr }}=43 \mathrm{meV}, J=69 \mathrm{meV}$ for PL-dimer and $J=50 \mathrm{meV}$ for P1- and P2-dimers). Delocalized charge carriers explain the observed efficient band-like charge transport with high $\mu_{\mathrm{e}}$ in this material. ${ }^{10}$ Disruption of charge delocalization by intermolecular vibrations explains the $\mu_{\mathrm{e}}$ decrease with temperature rise. In contrast, for TCNQ the delocalization criterion is not fulfilled. Assuming $d=1$ yields $J_{\text {cr }}=125 \mathrm{meV}$, while $J_{\max }=69 \mathrm{meV}$, i.e. $J_{\max }<J_{\mathrm{cr}}$. Even assuming $d=3$, we obtain $J_{\mathrm{cr}}=42 \mathrm{meV}$, but in this case $J>J_{\mathrm{cr}}$ for only one direction (P1), indicating the invalidity of the assumption. Therefore, the charges are localized in TCNQ resulting in hopping charge transport mechanism, which corresponds to the experiment. In addition, lower $d$ results in a stronger impact of static disorder (defects) and dynamic disorder (thermal fluctuations), which are inevitable in OSCs at room temperature and also promote charge localization. ${ }^{3,11}$ In the case of (quasi)1D transport along the stacks, a single defect in the stack can damage the transport pathway, while in the case of 3D transport, charges can easily circumvent the obstacle..$^{30-32}$ Summing up, the dimensionality of intermolecular charge delocalization explains the outstanding band-like electron transport in the $\mathrm{F}_{2}$-TCNQ crystal and inefficient hopping transport in TCNQ, which was the second issue addressed in the study.

An improvement in the charge transport with dimensionality for structurally close materials was observed in a number of studies. $^{9,33}$ In the series of crystalline dinaphtho[2,3-b:2', $\left.3^{\prime}-d\right]$ thiophene derivatives, ${ }^{34}$ the mobility increased 4 times for materials with an additional transport direction with $J>k T$. For crystalline $6 H$-pyrrolo[3,2- $\left.b: 4,5-b^{\prime}\right]$ bis $[1,4]$ benzothiazine derivatives, ${ }^{35}$ $\mu$ was significantly larger in the crystals with several transport directions and moderate $J$ than in the crystals with a single transport direction and large $J$. Within the series of bulky endcapped [1]benzothieno[3,2- $b]$ benzothiophene (BTBT) crystals, compounds with $J>2 k T$ in several directions exhibited $\mu$ about two orders of magnitude larger than the other materials. ${ }^{36}$ For the crystals of didodecyl-BTBT isomers, an unprecedented $\mu$ was observed for the compound with $2 \mathrm{D}$ transport and $J_{\max } \sim 60 \mathrm{meV}$, while the compound with an extremely large $J_{\max }=129 \mathrm{meV}$ but 1D transport exhibited a 340-fold lower $\mu .{ }^{37}$ Moreover, the same trend of $\mu$ increase with charge transport dimensionality was observed for arrays of nanoparticles. ${ }^{38}$ Noteworthily, the hopping model was unable to describe the data from ref. 37 even qualitatively, and only the band model considering charge delocalization reproduced the strong positive impact of charge transport dimensionality on $\mu$.

Our results extend far beyond the $\mathrm{F}_{n}$-TCNQ crystal series. We anticipate that intermolecular charge delocalization is especially important for OSCs consisting of small-sized conjugated molecules (e.g. derivatives of naphthalene-diimide (NDI), tetrathiafulvalene (TTF), etc.). These molecules have large $\lambda$, and intermolecular delocalization with high dimensionality is required for efficient charge transport according to our findings. In OSCs consisting of large conjugated molecules like tetracene, rubrene, pentacene, dinaphtho-thieno-thiophene (DNTT), etc., significant charge delocalization occurs already within the molecules, resulting in low $\lambda$ and making the intermolecular delocalization (especially in the direction of the long axis) less important.

Finally, the obtained results show that the frequently used screening of OSCs based just on single molecule properties, or 
accounting only the RIM-induced $\lambda$ decrease in the crystal (e.g. that provided in ref. 21), can overlook OSCs built from molecules of small size. For instance, this approach could not predict high mobility in $\mathrm{F}_{2}$-TCNQ in principle. In order to improve the screening method, intermolecular charge delocalization and the effect of dimensionality should be considered, at least at a very simple level provided by eqn (6).

\section{Conclusions}

Electron transport in the $\mathrm{F}_{n}$-TCNQ $(n=0,2,4)$ crystal family was investigated theoretically using DFT and QM/MM approaches. We have shown that in the TCNQ and F $_{2}$-TCNQ crystals, intermolecular charge delocalization can reduce dramatically the intramolecular reorganization energy, providing an opportunity for efficient band-like charge transport. However, while in $\mathrm{F}_{2}$-TCNQ large charge transfer integrals promote three-dimensional charge delocalization resistant to vibrations and defects, (quasi)onedimensional delocalization in TCNQ is readily destroyed by the latter, resulting in inefficient hopping charge transport. We suggest that intermolecular charge delocalization and its high dimensionality are important also for charge transport in other materials consisting of small-sized molecules, and current guidelines for high-mobility organic semiconductors should be corrected by considering these two factors.

\section{Conflicts of interest}

There are no conflicts to declare.

\section{Acknowledgements}

The author would like to thank D. Paraschuk, V. Bruevich, M. Vener and I. Chernyshov for thoughtful critical discussions and ideas. The work was supported by the Russian Foundation for Basic Research (RFBR), projects \#16-32-60204 mol_a_dk and \#17-02-00841.

\section{References}

1 O. Ostroverkhova, Chem. Rev., 2016, 116, 13279-13412.

2 G. Schweicher, Y. Olivier, V. Lemaur and Y. H. Geerts, Isr. J. Chem., 2014, 54, 595-620.

3 S. Fratini, D. Mayou and S. Ciuchi, Adv. Funct. Mater., 2016, 26, 2292-2315.

4 Y. Li, V. Coropceanu and J.-L. Brédas, in The WSPC Reference on Organic Electronics: Organic Semiconductors, ed. J.-L. Brédas and S. R. Marder, World Scientific, Singapore, 2016, ch. 7, pp. 193-230.

5 C. Wang, H. Dong, W. Hu, Y. Liu and D. Zhu, Chem. Rev., 2012, 112, 2208-2267.

6 H. Li, L. Duan, D. Zhang and Y. Qiu, J. Phys. Chem. C, 2014, 118, 14848-14852.

7 Q. Guo, L. Wang, F. Bai, Y. Jiang, J. Guo, B. Xu and W. Tian, RSC Adv., 2015, 5, 18875-18880.
8 S. T. Bromley, F. Illas and M. Mas-Torrent, Phys. Chem. Chem. Phys., 2008, 10, 121-127.

9 I. Yavuz, B. N. Martin, J. Park and K. N. Houk, J. Am. Chem. Soc., 2015, 137, 2856-2866.

10 Y. Krupskaya, M. Gibertini, N. Marzari and A. F. Morpurgo, Adv. Mater., 2015, 27, 2453-2458.

11 I. Y. Chernyshov, M. V. Vener, E. V. Feldman, D. Y. Paraschuk and A. Y. Sosorev, J. Phys. Chem. Lett., 2017, 8, 2875-2880.

12 T. P. Nguyen, J. H. Shim and J. Y. Lee, J. Phys. Chem. C, 2015, 119, 11301-11310.

13 B. Baumeier, J. Kirkpatrick and D. Andrienko, Phys. Chem. Chem. Phys., 2010, 12, 11103-11113.

14 J. Kirkpatrick, Int. J. Quantum Chem., 2008, 108, 51-56.

15 H. Kobayashi, N. Kobayashi, S. Hosoi, N. Koshitani, D. Murakami, R. Shirasawa, Y. Kudo, D. Hobara, Y. Tokita and M. Itabashi, J. Chem. Phys., 2013, 139, 8.

16 A. Warshel and M. Levitt, J. Mol. Biol., 1976, 103, 227-249.

17 R. A. Marcus and N. Sutin, Biochim. Biophys. Acta, 1985, 811, 265-322.

18 W. Q. Deng and W. A. Goddard, J. Phys. Chem. B, 2004, 108, 8614-8621.

19 A. N. Sokolov, S. Atahan-Evrenk, R. Mondal, H. B. Akkerman, R. S. Sánchez-Carrera, S. Granados-Focil, J. Schrier, S. C. B. Mannsfeld, A. P. Zoombelt, Z. Bao and A. Aspuru-Guzik, Nat. Commun., 2011, 2, 437.

20 M. V. Basilevsky, A. V. Odinokov and K. G. Komarova, J. Phys. Chem. B, 2015, 119, 7430-7438.

21 C. Schober, K. Reuter and H. Oberhofer, J. Phys. Chem. Lett., 2016, 7, 3973-3977.

22 M. W. Schmidt, K. K. Baldridge, J. A. Boatz, S. T. Elbert, M. S. Gordon, J. H. Jensen, S. Koseki, N. Matsunaga, K. A. Nguyen, S. Su, T. L. Windus, M. Dupuis and J. A. Montgomery, J. Comput. Chem., 1993, 14, 1347-1363.

23 M. S. Gordon and M. W. Schmidt, in Theory and Applications of Computational Chemistry: the First Forty Years, ed. C. E. Dykstra, G. Frenking, K. S. Kim and G. E. Scuseria, Elsevier, Amsterdam, 2005, pp. 1167-1189.

24 M. Valiev, E. J. Bylaska, N. Govind, K. Kowalski, P. Straatsma, H. J. J. van Dam, D. Wang, J. Nieplocha, E. Apra, T. L. Windus and W. A. de Jong, Comput. Phys. Commun., 2010, 181, 1477-1489.

25 V. Coropceanu, R. S. Sánchez-Carrera, P. Paramonov, G. M. Day and J.-L. Brédas, J. Phys. Chem. C, 2009, 113, 4679-4686.

26 S. Illig, A. S. Eggeman, A. Troisi, L. Jiang, C. Warwick, M. Nikolka, G. Schweicher, S. G. Yeates, Y. Henri Geerts, J. E. Anthony and H. Sirringhaus, Nat. Commun., 2016, 7, 10736.

27 N. G. Martinelli, Y. Olivier, S. Athanasopoulos, M.-C. Ruiz Delgado, K. R. Pigg, D. A. da Silva Filho, R. S. SánchezCarrera, E. Venuti, R. G. Della Valle, J.-L. Brédas, D. Beljonne and J. Cornil, ChemPhysChem, 2009, 10, 2265-2273.

28 Y. Jiang, X. Zhong, W. Shi, Q. Peng, H. Geng, Y. Zhao and Z. Shuai, Nanoscale Horiz., 2016, 1, 53-59.

29 S. Larsson and A. Klimkans, Mol. Cryst. Liq. Cryst., 2001, 355, 217-229.

30 P. J. Skabara, J. B. Arlin and Y. H. Geerts, Adv. Mater., 2013, 25, 1948-1954. 
31 N. E. Jackson, K. L. Kohlstedt, L. X. Chen and M. A. Ratner, J. Chem. Phys., 2016, 145, 204102.

32 T. Vehoff, B. Baumeier, A. Troisi and D. Andrienko, J. Am. Chem. Soc., 2010, 132, 11702-11708.

33 H. Sirringhaus, T. Sakanoue and J.-F. Chang, Phys. Status Solidi B, 2012, 249, 1655-1676.

34 T. Okamoto, C. Mitsui, M. Yamagishi, K. Nakahara, J. Soeda, Y. Hirose, K. Miwa, H. Sato, A. Yamano, T. Matsushita, T. Uemura and J. Takeya, Adv. Mater., 2013, 25, 6392-6397.

35 Z. Wei, W. Hong, H. Geng, C. Wang, Y. Liu, R. Li, W. Xu, Z. Shuai, W. Hu, Q. Wang and D. Zhu, Adv. Mater., 2010, 22, 2458-2462.
36 G. Schweicher, V. Lemaur, C. Niebel, C. Ruzié, Y. Diao, O. Goto, W.-Y. Lee, Y. Kim, J.-B. Arlin, J. Karpinska, A. R. Kennedy, S. R. Parkin, Y. Olivier, S. C. B. Mannsfeld, J. Cornil, Y. H. Geerts and Z. Bao, Adv. Mater., 2015, 27, 3066-3072.

37 Y. Tsutsui, G. Schweicher, B. Chattopadhyay, T. Sakurai, J.-B. Arlin, C. Ruzié, A. Aliev, A. Ciesielski, S. Colella, A. R. Kennedy, V. Lemaur, Y. Olivier, R. Hadji, L. Sanguinet, F. Castet, S. Osella, D. Dudenko, D. Beljonne, J. Cornil, P. Samori, S. Seki and Y. H. Geerts, Adv. Mater., 2016, 28, 7106-7114.

38 Y. Wang, C. Duan, L. M. Peng and J. H. Liao, Sci. Rep., 2014, 4, 7565 . 